\title{
High-Grade Ovarian Serous Carcinoma Presenting as Androgenetic Alopecia
}

\author{
Anna Eversman, BS; Elisabeth Tracey, MD; Christine B. Warren, MD, MS
}

\section{PRACTICE POINTS}

- Laboratory assessment for possible androgen excess should be performed in patients with female pattern hair loss and include baseline serum total testosterone and dehydroepiandrosterone sulfate.

- Rapid onset or worsening of clinical hyperandrogenism should raise suspicion of malignancy.

- Transvaginal ultrasonography and possible pelvic magnetic resonance imaging are indicated for patients with clinical hyperandrogenism and an isolated testosterone level elevation.

To the Editor:

Female pattern hair loss is common, and the literature suggests that up to $56 \%$ of women experience hair thinning in their lifetime, with increased prevalence in older women. ${ }^{1}$ Pathophysiology is incompletely understood and involves the nonscarring progressive miniaturization of hair follicles, causing decreased production of terminal hairs relative to more delicate vellus hairs. Because vellus hairs have a shorter anagen growth phase than terminal hairs, hair loss is expedited. Androgen excess, when present, hastens the process by inducing early transition of hair follicles from the anagen phase to the senescent telogen phase. Serum testosterone levels are within reference range in most female patients with hair loss, suggesting the presence of additional contributing factors. ${ }^{2}$

Given the high prevalence of female pattern hair loss and the harm of overlooking androgen excess and an androgen-secreting neoplasm, dermatologists must recognize indications for further evaluation. Additional signs of hyperandrogenism, such as menstrual irregularities, acne, hirsutism, anabolic appearance, voice deepening, and clitoromegaly, are reasons for concern. ${ }^{3}$ Elevated serum androgen levels also should raise suspicion of malignancy. Historically, a total testosterone level above $200 \mathrm{ng} / \mathrm{dL}$ or a dehydroepiandrosterone sulfate (DHEA-S) level greater than $700 \mu \mathrm{g} / \mathrm{dL}$ prompted evaluation for a tumor. ${ }^{4}$ More recent studies show that tumorinduced increases in serum androgen levels are highly variable, challenging the utility of these cutoffs. ${ }^{5}$

A 70-year-old woman presented with hair loss over the last 12 years with accentuated thinning on the frontal and vertex scalp. The patient's primary care physician previously made a diagnosis of androgenetic alopecia and recommended topical minoxidil. Although the patient had a history of excess facial and body hair since young adulthood, she noted a progressive increase in the density of chest and back hair, prominent coarsening of the texture of the facial and body hair, and new facial acne in the last 3 years. Prior to these changes, the density and texture of the scalp and body hair had been stable for many years.

Ms. Eversman and Dr. Warren are from the Cleveland Clinic Lerner College of Medicine, Case Western Reserve University, Ohio. Dr. Warren also is from and Dr. Tracey is from the Department of Dermatology, Cleveland Clinic.

The authors report no conflict of interest.

Correspondence: Christine B. Warren, MD, MS, Department of Dermatology, Cleveland Clinic, 9500 Euclid Ave A61, Cleveland, OH 44195 (warrenc@ccf.org).

doi:10.12788/cutis.0151 
Although other postmenopausal females in the patient's family displayed patterned hair loss, they did not possess coarse and dense hair on the face and trunk. Her family history was notable for ovarian cancer in her mother (in her 70s) and breast cancer in her maternal grandmother (in her 80s).

A review of systems was notable only for decreased energy. Physical examination revealed a well-appearing older woman with coarse terminal hair growth on the cheeks, submental chin, neck, chest, back, and forearms. Scalp examination indicated diffusely decreased hair density, most marked over the vertex, crown, and frontal scalp, without scale, erythema, or loss of follicular ostia (Figure 1).

Laboratory evaluation revealed elevated levels of total testosterone (106 ng/dL [reference range, $<40 \mathrm{ng} / \mathrm{dL}$ ]) and free testosterone $(32.9 \mathrm{pg} / \mathrm{mL}$ [reference range, $1.8-10.4 \mathrm{pg} / \mathrm{mL}])$ but a DHEA-S level within reference range, suggesting an ovarian source of androgen excess. The CA-125 level was elevated ( $89 \mathrm{U} / \mathrm{mL}$ [reference range, $<39 \mathrm{U} / \mathrm{mL}])$.

Pelvic ultrasonography was suspicious for an ovarian pathology. Follow-up pelvic magnetic resonance imaging (MRI) demonstrated a $2.5-\mathrm{cm}$ mass abutting the left ovary (Figure 2). The patient was given a diagnosis of stage IIIA high-grade ovarian serous carcinoma with lymph node involvement. Other notable findings from the workup included a BRCA2 mutation and concurrent renal cell carcinoma. After bilateral salpingo-oophorectomy, partial nephrectomy, and chemotherapy with carboplatin and paclitaxel, the testosterone level returned to within reference range and remained stable for the next 2 years of follow-up.

Female pattern hair loss is common in postmenopausal women and is a frequent concern in patients

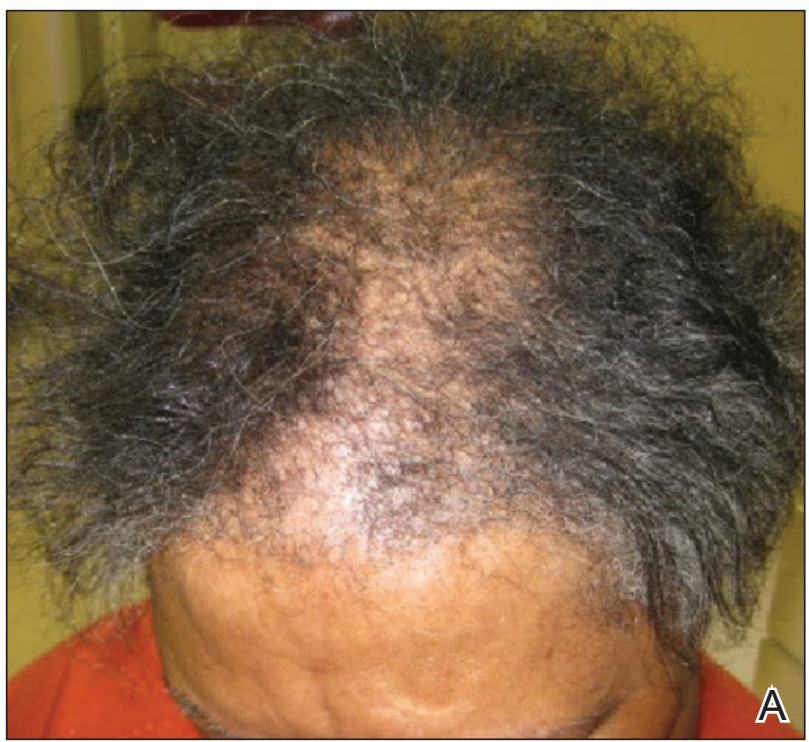

presenting to dermatology. Although most cases of androgenetic alopecia are isolated or secondary to benign conditions, such as polycystic ovary syndrome or nonclassic congenital adrenal hyperplasia, a small minority ( $<1 \%$ of women presenting with signs of hyperandrogenism) have an androgen-secreting tumor. ${ }^{6}$

Rapid onset or worsening of clinical hyperandrogenism, as seen in our patient, should raise concern for pathology; serum total testosterone and DHEA-S levels should be evaluated. Abnormally elevated serum androgens are associated with malignancy; however, there is variability in the recommended cutoff levels to prompt suspicion for an androgen-producing tumor and further workup in postmenopausal women. In the case of testosterone elevation, classic teaching designates a testosterone level greater than $200 \mathrm{ng} / \mathrm{dL}$ as the appropriate threshold for concern, but this level is now debated. In a series of women with hyperandrogenism referred to a center for suspicion of an androgen-secreting tumor, those with a tumor had, on average, a significantly higher $(260 \mathrm{ng} / \mathrm{dL})$ testosterone level than women who had other causes $(90 \mathrm{ng} / \mathrm{dL})(P<.05) .{ }^{6}$ The authors of that study proposed a cutoff of $1.4 \mathrm{ng} / \mathrm{mL}$ because women in their series who had a tumor were 8.4 times more likely to have a testosterone level of $1.4 \mathrm{ng} / \mathrm{mL}$ or higher than women without a tumor. However, this cutoff was only $92 \%$ sensitive and $70 \%$ specific. ${ }^{6}$ The degree of androgen elevation is highly variable in both tumorous and benign pathologies with notable overlap, challenging the notion of a clear cutoff.

Imaging is indicated for a patient presenting with both clinical and biochemical hyperandrogenism. Patients with an isolated testosterone level elevation can be evaluated with transvaginal ultrasonography; however, detection

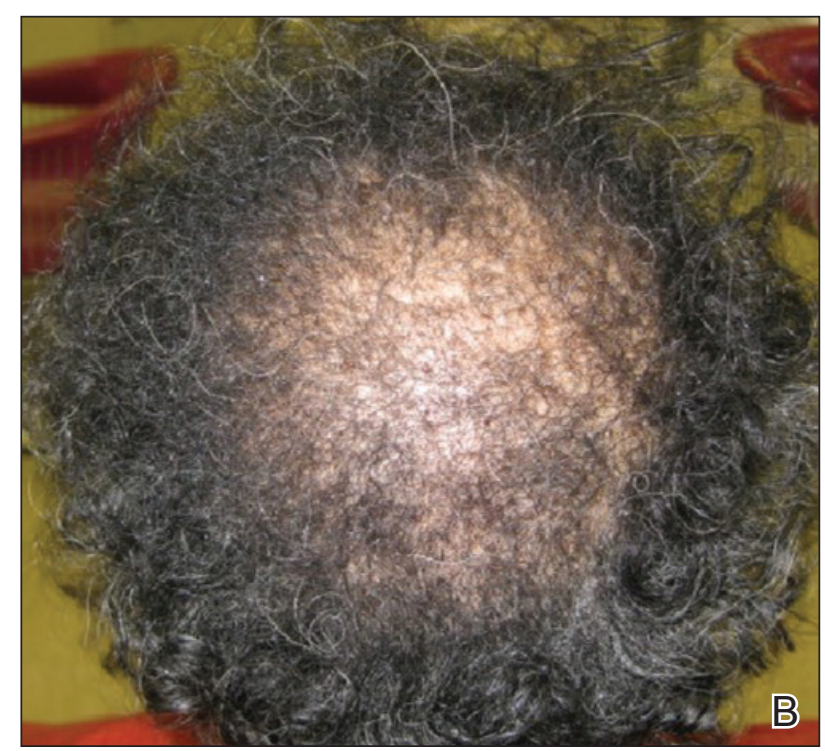

FIGURE 1. A and B, Diffusely decreased hair density, most marked over the vertex, crown, and frontal scalp, without scale, erythema, or loss of follicular ostia. 

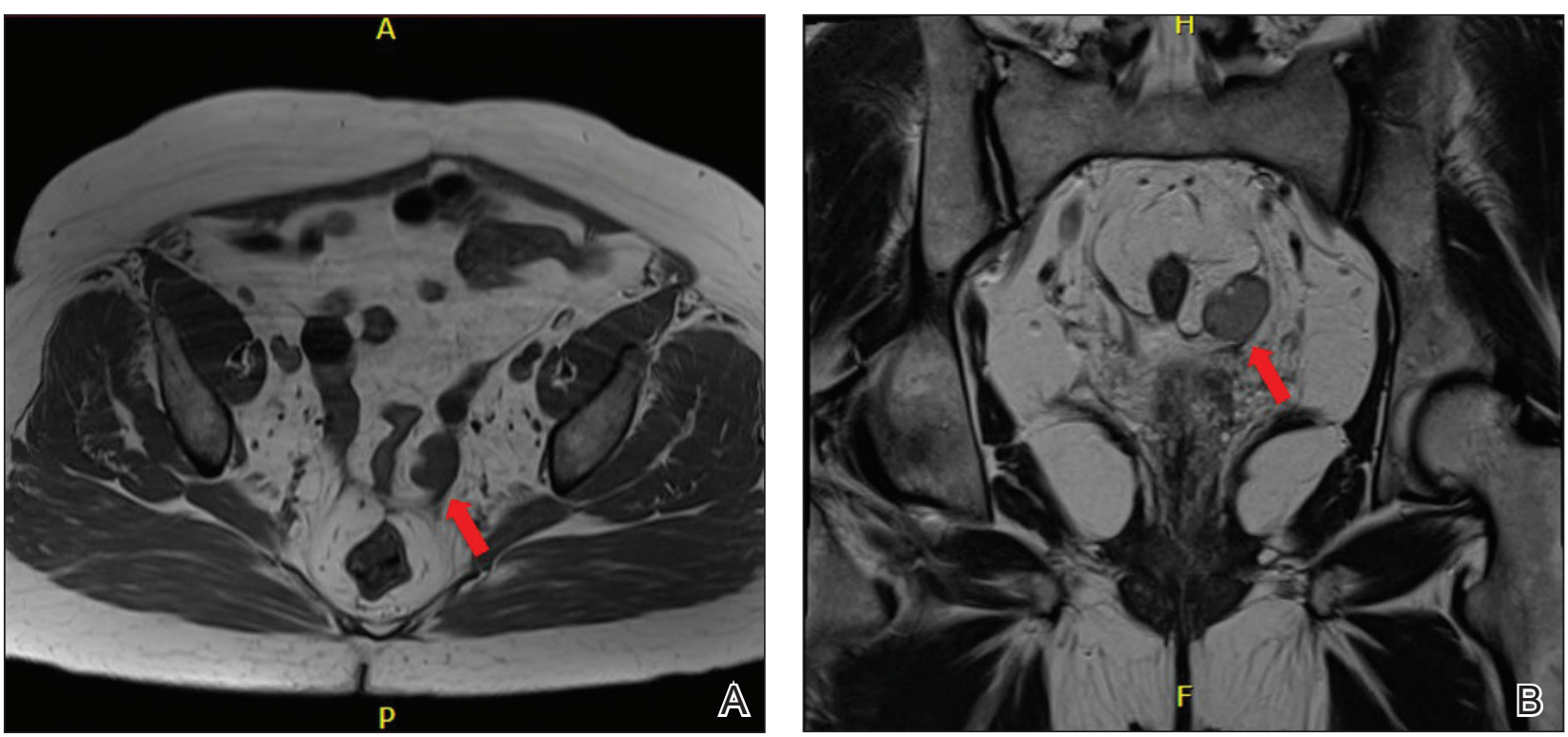

FIGURE 2. A and B, Axial T1-weighted and sagittal T2-weighted pelvic magnetic resonance imaging, respectively, demonstrated a 2.5-cm mass (red arrows) abutting the left ovary.

and characterization of malignancies is highly dependent on the skill of the examiner. ${ }^{8,9}$ The higher sensitivity and specificity of pelvic MRI reduces the likelihood of missing a malignancy and unnecessary surgery. Tumors too small to be visualized by MRI rarely are malignant. ${ }^{10}$

Sex cord-stromal cell tumors, despite representing fewer than $10 \%$ of ovarian tumors, are responsible for the majority of androgen-secreting malignancies. Our patient presented with clinical hyperandrogenism with an elevated testosterone level in the setting of a serous ovarian carcinoma, which is an epithelial neoplasm. Epithelial tumors are the most common type of ovarian tumor and typically are nonfunctional, though they have been reported to cause hyperandrogenism through indirect mechanisms. It is thought that both benign and malignant epithelial tumors can induce stromal hyperplasia or luteinization, leading to an increase in androgen levels. ${ }^{6}$

Due to the high prevalence of androgenetic alopecia and hirsutism in aging women, identification of androgen-secreting neoplasms by clinical presentation is challenging. A wide range of serum testosterone levels is possible at presentation, which complicates diagnosis. This case highlights the importance of correlating clinical and biochemical hyperandrogenism in raising suspicion of malignancy in older women presenting with hair loss.

\section{REFERENCES}

1. Carmina E, Azziz R, Bergfeld W, et al. Female pattern hair loss and androgen excess: a report from the multidisciplinary androgen excess and PCOS committee. J Clin Endocrinol Metab. 2019;104:2875-2891.

2. Herskovitz I, Tosti A. Female pattern hair loss. Int J Endocrinol Metab. 2013;11:e9860.

3. Rothman MS, Wierman ME. How should postmenopausal androgen excess be evaluated? Clin Endocrinol (Oxf). 2011;75:160-164.

4. Derksen J, Nagesser SK, Meinders AE, et al. Identification of virilizing adrenal tumors in hirsute women. N Engl J Med. 1994;331:968-973.

5. Kaltsas GA, Isidori AM, Kola BP, et al. The value of the low-dose dexamethasone suppression test in the differential diagnosis of hyperandrogenism in women. J Clin Endocrinol Metab. 2003; 88:2634-2643.

6. Sarfati J, Bachelot A, Coussieu C, et al; Study Group Hyperandrogenism in Postmenopausal Women. Impact of clinical, hormonal, radiological, immunohistochemical studies on the diagnosis of postmenopausal hyperandrogenism. Eur J Endocrinol. 2011;165:779-788.

7. Glintborg D, Altinok ML, Petersen KR, et al. Total testosterone levels are often more than three times elevated in patients with androgensecreting tumours. BMJ Case Rep. 2015;2015:bcr2014204797.

8. Iyer VR, Lee SI. MRI, CT, and PET/CT for ovarian cancer detection and adnexal lesion characterization. AJR Am J Roentgenol. 2010; 194:311-321.

9. Rauh-Hain JA, Krivak TC, Del Carmen MG, et al. Ovarian cancer screening and early detection in the general population. Rev Obstet Gynecol. 2011;4:15-21.

10. Horta M, Cunha TM. Sex cord-stromal tumors of the ovary: a comprehensive review and update for radiologists. Diagn Interv Radiol. 2015;21:277-286. 